\title{
Balance of Dark and Luminous Mass in Rotating Galaxies
}

\author{
Stacy S. McGaugh \\ Department of Astronomy, University of Maryland, College Park, Maryland 20742-2421, USA \\ (Received 2 March 2005; revised manuscript received 29 August 2005; published 20 October 2005)
}

\begin{abstract}
A fine balance between dark and baryonic mass is observed in spiral galaxies. As the contribution of the baryons to the total rotation velocity increases, the contribution of the dark matter decreases by a compensating amount. This poses a fine-tuning problem for $\Lambda$ CDM galaxy formation models, and may point to new physics for dark matter particles or even a modification of gravity.
\end{abstract}

PACS numbers: 95.35.+d, 98.52.Nr, 98.62.Ck, 98.62.Dm

The rotation curves of spiral galaxies become approximately flat at the largest radii observed [1,2]. This is one of the strongest indications of the need for dynamically dominant dark matter in the Universe. While the need for dark matter at large radii is clear, its quantity and distribution is less so.

Cogent but contradictory arguments can be made about the relative importance of dark and luminous mass at small radii in spirals. The inner shape of rotation curves is well predicted by the distribution of observed baryons [3,4], implying that galactic disks are maximal in the sense that they contribute the bulk of the mass at small radii. On the other hand, measurements of the velocity dispersions of stars perpendicular to galactic disks [5,6] often suggest submaximal disks.

Rotation curve shapes measured for spiral galaxies are found to correlate strongly with the observed luminosity distribution [7-10]. There appears to be a characteristic acceleration scale $[11,12]$ at which the baryons alone can no longer account for the observed dynamics. The amplitude of the mass discrepancy correlates with acceleration, providing accurate, albeit empirically motivated, determinations of the baryonic masses [11] of spiral galaxies.

This is significant progress in disentangling the dark and baryonic mass. However, the physics behind these empirical relations is unclear. Here I examine the balance between dark and luminous mass in spirals, and outline the possible interpretations.

The data used here are tabulated in Ref. [13]. This is a sample of 60 galaxies for which we have extensive, detailed knowledge. In particular, their baryonic masses, the sum of stars and gas

$$
\mathcal{M}_{b}=\mathcal{M}_{*}+\mathcal{M}_{g},
$$

are well determined. Examples of the rotation curves of two galaxies are shown in Fig. 1.

Galaxy structural parameters describing the distribution of baryons are essential to the analysis here. The azimuthally averaged light distribution of spiral galaxies can often be approximated as an exponential disk

$$
\Sigma(R)=\Sigma_{0} e^{-R / R_{d}},
$$

where $\Sigma_{0}$ is the central surface brightness and $R_{d}$ is the scale length of the stellar disk. Such a mass distribution has a rotation curve [14] that peaks at $2.2 R_{d}$. In a system with both stars and dark matter, an interesting measure is the velocity $V_{2.2}$ at this point [15-17] where the mass of the stars is most relevant.

Gravity makes no distinction between stars and gas, so a preferable measure is $V_{p}$, the velocity at the radius $R_{p}$ where the sum of these baryonic components make their maximum contribution to the total rotation. For star dominated galaxies, there is little difference between these quantities. For gas rich galaxies, which are well represented here, $R_{p}$ can be considerably larger than $2.2 R_{d}$. $V_{p}$ usually differs little from $V_{2.2}$ because of the near flatness of rotation curves, but in some cases there are significant if modest differences.

To include the gas as well as the stars, I use the masses tabulated in Ref. [13] and measure $R_{p}$ and $V_{p}$ for each galaxy. $R_{p}$ is taken from the combined surface density map (Fig. 1); no assumption is made that the disk is purely exponential. It is useful to define a baryonic surface mass density

$$
\Sigma_{b}=\frac{3 \mathcal{M}_{b}}{4 R_{p}^{2}}
$$

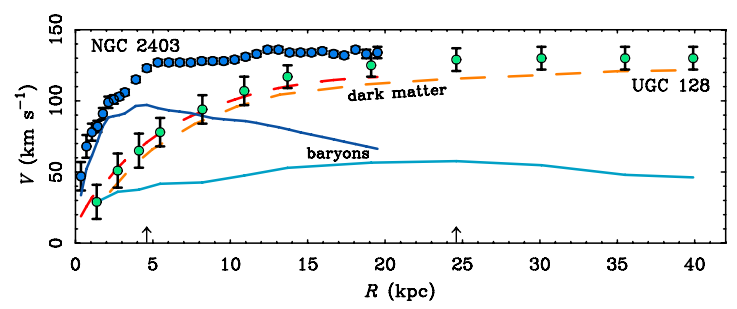

FIG. 1 (color online). The rotation curves of two galaxies, NGC 2403 and UGC 128, of similar mass but very different size. The contributions of the baryonic components (stars plus gas: solid lines) are very different even though the dark matter halos (dashed lines) are similar. Lines for the baryons and dark halo of NGC 2403 end with its velocity data at $20 \mathrm{kpc}$ while those of UGC 128 continue to $40 \mathrm{kpc}$. Arrows mark the radius $R_{p}$ where the peak of the baryonic rotation occurs. 
This is equivalent to $\Sigma_{0}$ for a purely exponential disk with $R_{p}=2.2 R_{d}$.

The size and rotation velocity of disks correlates with their baryonic mass (Fig. 2). The mass-velocity relation is sometimes referred to as the baryonic Tully-Fisher relation [18]. A number of variations [19,20] making use of different mass and velocity estimators exist. The critical difference here from Ref. [13] is the consideration of $V_{p}$ as the velocity measure as well as the asymptotic flat velocity $V_{f}$. It is of interest to find how much the baryons contribute to $V_{p}$, so $V_{b}\left(R_{p}\right)$ is also illustrated in Fig. 2 for the adopted baryonic mass. Two size estimators are also shown: the scale length $R_{d}$ of the stars and the radius of the peak of the baryonic rotation $R_{p} . R_{d}$ is the quantity traditionally used [15-17] when information about the gas is not available, and is shown for comparison to the size $R_{p}$ used here. Fits to the data of the form $\log y=a+b \log \mathcal{M}_{b}$ are given in Table I.

A remarkable aspect of the Tully-Fisher [21] relation (the precursor to the mass-velocity relation) is that it shows no variation with scale size [22-24]. It is as if the distribution of luminous mass has no impact on the global dynamics [19,25]. Reference [15] proposed using the velocity-scale length residuals as a measure of the relative importance of baryonic disk and dark matter halo. The residual from the fitted relations for each object $i$ is $\delta y_{i} \equiv y_{i}-y\left(\mathcal{M}_{d, i}\right)$.

The idea is simple: if the luminous disk contributes significantly to the total mass, variations in the distribution of baryons should affect the observed velocity. Imagine
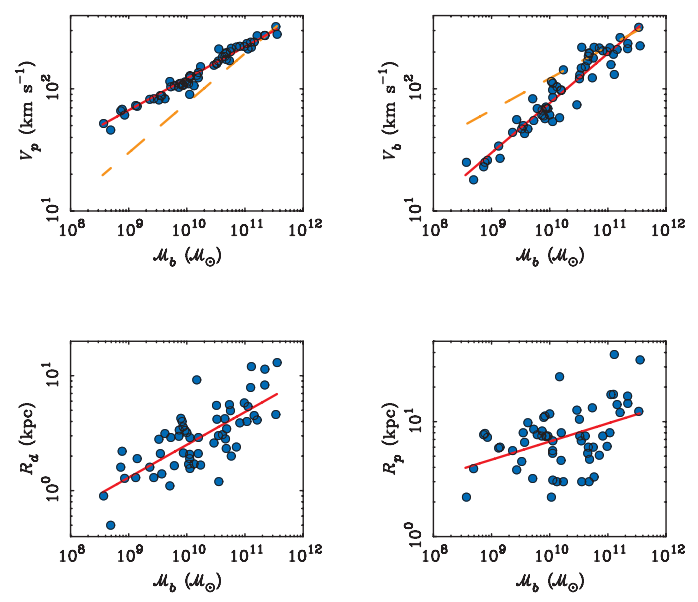

FIG. 2 (color online). Correlations of various galaxy properties with baryonic mass. Mass is measured in solar masses, velocities in $\mathrm{km} \mathrm{s}^{-1}$, and radii in kpc. Top left: the observed velocity $V_{p}$ at $R_{p}$. Top right: the velocity $V_{b}$ due to the baryons at $R_{p}$. Bottom left: the scale length of the stellar disk $R_{d}$. Bottom right: the radius $R_{p}$ where the baryons contribute maximally to the rotation. The solid lines are the fits to the data given in Table I. For comparison, the fits to the other velocity is shown as a dashed line in the top panels.
TABLE I. Correlations with baryonic mass.

\begin{tabular}{ccc}
\hline \hline$y^{\mathrm{a}}$ & $a$ & $b$ \\
\hline$V_{p}$ & $-0.52 \pm 0.03$ & $0.26 \pm 0.01$ \\
$V_{f}$ & $-0.38 \pm 0.05$ & $0.25 \pm 0.01$ \\
$V_{b}$ & $-2.19 \pm 0.18$ & $0.41 \pm 0.02$ \\
$R_{d}$ & $-2.45 \pm 0.35$ & $0.29 \pm 0.03$ \\
$R_{p}$ & $-0.75 \pm 0.44$ & $0.16 \pm 0.04$ \\
\hline \hline
\end{tabular}

${ }^{\text {a}}$ Relations of the form $\log _{10} y=a+b \log _{10} \mathcal{M}_{b}$.

taking a compact, high surface brightness galaxy and stretching it out to become a diffuse, low surface brightness galaxy. This is essentially what is illustrated in Fig. 1. Since $V^{2} \propto \mathcal{M} / R$, the velocity $V_{b}$ attributable to the baryonic component must decline as $R_{p}$ grows at fixed mass.

A family of pure disks devoid of dark matter should have $V_{p}=V_{b}$ and obey a residual relation [15]

$$
\frac{\partial \log V_{p}}{\partial \log R_{p}}=-\frac{1}{2}
$$

For a combined system of baryonic disk plus dark matter halo, the observed slope will depend on the halo model and the degree of disk contribution. Residuals from both the luminosity-velocity (Tully-Fisher) and mass-velocity (baryonic Tully-Fisher) relations are shown in Fig. 3. Luminosities measured in the $B$ band are available for all galaxies. $K^{\prime}$-band photometry [19] is available for 24 of the 60 galaxies. This near-infrared $(2.2 \mu \mathrm{m})$ pass band is thought to give the closest mapping between stellar light and mass [26], providing a check that the results are not specific to the particular choice of mass estimator.

No residuals of the type expected for a pure disk are observed. Indeed, the $B$ and $K^{\prime}$ bands have slopes of opposite sign, though neither is significantly different from zero (Table II). The residuals from the mass-flat
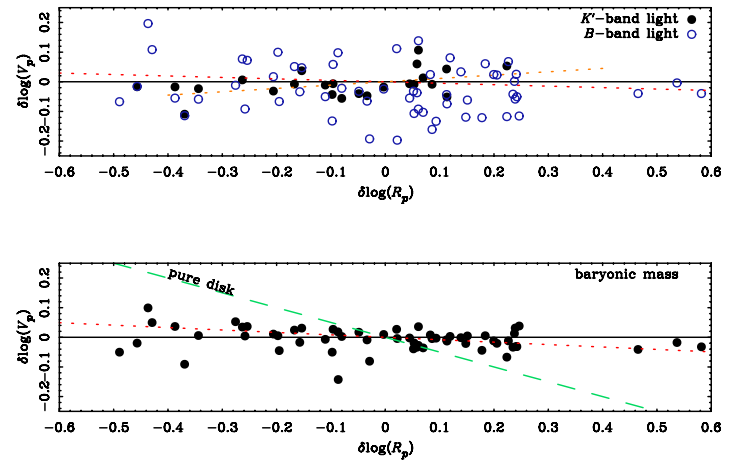

FIG. 3 (color online). Velocity residuals from the luminosity- $V_{p}$ relation (top) and the $\mathcal{M}_{b}-V_{p}$ relation (bottom) as a function of scale length residuals. In the top panel, open circles are $B$-band data and filled circles are $K^{\prime}$-band data. Fits to these residuals (dotted lines) have slopes close to zero (solid lines). The mass residuals do not show the slope expected for pure disks (dashed line). 
TABLE II. Residual slopes.

\begin{tabular}{lcc}
\hline \hline Estimator & $\partial \log V_{p} / \partial \log R_{p}$ & $\partial \log V_{f} / \partial \log R_{p}$ \\
\hline$K^{\prime}$ band & $+0.11 \pm 0.05$ & $+0.06 \pm 0.04$ \\
$B$ band & $-0.05 \pm 0.05$ & $+0.01 \pm 0.04$ \\
Mass & $-0.08 \pm 0.02$ & $-0.00 \pm 0.01$ \\
\hline \hline
\end{tabular}

rotation velocity relation have slope zero. When $V_{p}$ is used as the velocity measure instead of $V_{f}$, a slightly negative slope is inferred. This is marginally $(4 \sigma)$ different from zero. Given the nature of astronomical observations, it would be unwise to read too much into the particular value of the slope. What is clear is that galaxies do not follow the pure disk prediction, having instead a residual slope very near to zero.

A tempting interpretation is that all spiral galaxies are dominated by dark matter. There is little sensitivity to $R_{p}$ because the baryonic mass is negligible. We have estimates of the baryonic mass already, independent of this argument, so it is interesting to check if this is so.

We can directly compare the contribution of the baryonic $\left(V_{b}\right)$ and dark halo $\left(V_{h}\right)$ components to the total observed velocity $V_{p}$ at $R_{p}$. This is shown in Fig. 4 where it can be seen that the fractional contribution of the baryons, $V_{b} / V_{p}$, is well correlated with the baryonic surface density. This is not specific to the mass estimates adopted here, being clear also in the $K^{\prime}$-band data. For either case, roughly half of the sample is baryon dominated within $R_{p}$ $\left(V_{b}>V_{h}\right)$.

Clearly the small slope of the residuals in Fig. 3 does not require submaximal disks, as the situation may arise even when some disks are maximal. The specific relation between the baryon contribution and surface density (well fit
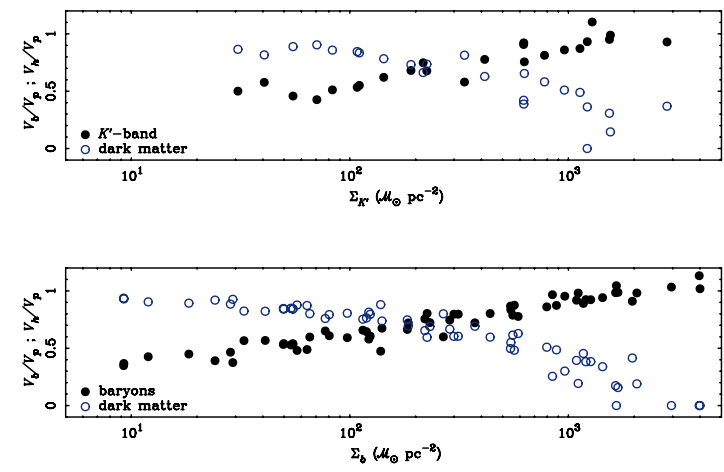

FIG. 4 (color online). The fractional contribution to the total velocity at the peak radius by baryons $\left(V_{b} / V_{p}\right)$ and dark matter $\left(V_{h} / V_{p}\right)$. These correlate with baryonic surface density, determined either from the $K^{\prime}$-band light [19] and stellar population models [26] (top) or Eq. (3) (bottom). Disks come closer to being maximal as surface density increases. Curiously, the dark matter contribution declines in compensation, maintaining a seesaw balance with the baryons. by $\left.V_{b} / V_{p}=0.05+0.28 \log \Sigma_{b}\right)$ depends on the mass estimator, but such a relation must always be present. For any choice of mass estimator, there is a fine-tuning between $V_{b}$ and $V_{h} . V_{p}^{2}=V_{b}^{2}+V_{h}^{2}$, yet $V_{p}$ hardly varies with the distribution of baryons, while $V_{b}$ must do so by definition. This leads to the seesaw balance between baryons and dark matter apparent in Fig. 4.

This balance constitutes a fine-tuning problem for $\Lambda \mathrm{CDM}$. One does not naively expect the baryonic and dark halo contributions to anticorrelate. In the most basic models $[27,28]$ one expects that when the baryons collapse to form the visible galaxy, they draw some of the dark matter along with them (an effect commonly referred to as adiabatic contraction [29-31]). In such a scenario, one would expect more baryons to mean more dark matter, not less.

The extent to which the observed balance constitutes a fine-tuning problem is open to debate [25,32]. Reference [32] suggests that feedback from the activity of massive stars - the kinetic energy from stellar winds and supernova explosions - may provide a mechanism to reproduce the observations. It seems strange to invoke such chaotic effects to impart an organization to the models which is not naturally there. Worse, a minority of the baryonic component must strongly affect the dominant dark matter - a case of the tail wagging the dog. In order to work, feedback must act in a very specific fashion which has yet to be realized in sophisticated numerical simulations [33,34]. A completely satisfactory theory of galaxy formation has yet to emerge.

It is worth noting that $\partial \log V_{f} / \partial \log R_{p}=0$ is an a priori prediction of an alternative to dark matter, the modified Newtonian dynamics (MOND) [12]. Indeed, while dark matter models struggle with the cusp/core problem [35], MOND fits the rotation curve data in considerable detail [9]. No fine-tuning is required.

MOND has had other predictions realized, for example, in the dynamics of low surface brightness galaxies [36] and in the peak amplitude ratio of the angular power spectrum of the cosmic background radiation [37,38]. A long standing theoretical objection to MOND has been the lack of a generally covariant theory, but this obstacle has recently been addressed [39]. This in turn may allow the theory to address issues like large scale structure [40] about which it has previously been mute.

While MOND has been surprisingly successful, it is not without problems. The most serious problem facing it at present is the residual mass discrepancy in clusters of galaxies. The MOND formula applied to these systems fails to explain the missing mass problem [41-44] as it does in individual galaxies. Perhaps this will prove fatal to the theory, or perhaps there is more conventional mass in clusters that remains undetected.

There is a third possibility. We still know very little about the nature of the dark matter (presuming it exists). 
It may possess some property that imparts the observed balance with baryons in galaxies. This idea implies a specific interaction between the two that is in some way repulsive: the greater the surface density of baryons, the less that of dark matter. Such a repulsion would help explain the apparent lack of dark matter in high density regions like globular clusters [45] and elliptical galaxies [46]. It would also have important implications for direct detection experiments.

No interaction with baryons of the sort envisaged is in the nature of most hypothesized dark matter candidates. Neither cold dark matter nor frequently discussed alternatives like warm $[47,48]$ or self-interacting [49] dark matter do anything of the sort. Whether it is even possible to endow dark matter with the appropriate properties [50] is difficult to say as the possibility has yet to be thoroughly explored.

Irrespective of which type of interpretation may seem preferable - the details of galaxy formation, modified dynamics, or baryon-repulsive dark matter-clearly there is important physics at work that has yet to be elucidated.

The work of S. S. M. is supported in part by NSF Grant No. AST0505956.

[1] V. C. Rubin, N. Thonnard, and W. K. Ford, Astrophys. J. 238, 471 (1980).

[2] A. Bosma, Astron. J. 86, 1791 (1981).

[3] T. S. van Albada and R. Sancisi, Phil. Trans. R. Soc. A 320, 447 (1986).

[4] P. Palunas and T. B. Williams, Astron. J. 120, 2884 (2000).

[5] R. Bottema, Astron. Astrophys. 275, 16 (1993).

[6] M. Kregel, P. C. van der Kruit, and K. C. Freeman, Mon. Not. R. Astron. Soc. 358, 503 (2005).

[7] V.C. Rubin, W. K. Ford, N. Thonnard, and D. Burstein, Astrophys. J. 261, 439 (1982).

[8] R. Sancisi, in IAU Symposium, Sydney, Australia, 2003, edited by S. D. Ryder, D. J. Pisano, M. A. Walker, and K. C. Freeman (Astronomical Society of the Pacific, San Francisco, 2004), p. 233.

[9] R.H. Sanders and S.S. McGaugh, Annu. Rev. Astron. Astrophys. 40, 263 (2002).

[10] M. Persic and P. Salucci, Astrophys. J. 368, 60 (1991).

[11] S. S. McGaugh, Astrophys. J. 609, 652 (2004).

[12] M. Milgrom, Astrophys. J. 270, 371 (1983).

[13] S.S. McGaugh, astro-ph/0506750 [Astrophys. J. (to be published)].

[14] K. C. Freeman, Astrophys. J. 160, 811 (1970).

[15] S. Courteau and H. Rix, Astrophys. J. 513, 561 (1999).

[16] A. A. Dutton, F. C. van den Bosch, S. Courteau, and A. Dekel, astro-ph/0501256.

[17] J. Pizagno et al., astro-ph/0504581.

[18] S. S. McGaugh, J.M. Schombert, G. D. Bothun, and W. J. G. de Blok, Astrophys. J. Lett. 533, L99 (2000).

[19] M. A. W. Verheijen, Astrophys. J. 563, 694 (2001).

[20] E. F. Bell and R. S. de Jong, Astrophys. J. 550, 212 (2001).
[21] R. B. Tully and J.R. Fisher, Astron. Astrophys. 54, 661 (1977).

[22] D. Sprayberry, G. M. Bernstein, C. D. Impey, and G. D. Bothun, Astrophys. J. 438, 72 (1995).

[23] M. A. Zwaan, J. M. van der Hulst, W. J. G. de Blok, and S. S. McGaugh, Mon. Not. R. Astron. Soc. 273, L35 (1995).

[24] G. L. Hoffman, E.E. Salpeter, B. Farhat, T. Roos, H. Williams, and G. Helou, Astrophys. J. Suppl. Ser. 105, 269 (1996).

[25] S. S. McGaugh and W. J. G. de Blok, Astrophys. J. 499, 41 (1998).

[26] E. F. Bell, D. H. McIntosh, N. Katz, and M. D. Weinberg, Astrophys. J. Suppl. Ser. 149, 289 (2003).

[27] J. J. Dalcanton, D. N. Spergel, and F. J. Summers, Astrophys. J. 482, 659 (1997).

[28] H. J. Mo, S. Mao, and S.D. M. White, Mon. Not. R. Astron. Soc. 295, 319 (1998).

[29] G. R. Blumenthal, S. M. Faber, R. Flores, and J. R. Primack, Astrophys. J. 301, 27 (1986).

[30] O. Y. Gnedin, A. V. Kravtsov, A. A. Klypin, and D. Nagai, Astrophys. J. 616, 16 (2004).

[31] J.A. Sellwood and S.S. McGaugh, astro-ph/0507589 [Astrophys. J. (to be published)].

[32] F. C. van den Bosch and J. J. Dalcanton, Astrophys. J. 534, 146 (2000).

[33] F. Governato, L. Mayer, J. Wadsley, J. P. Gardner, B. Willman, E. Hayashi, T. Quinn, J. Stadel, and G. Lake, Astrophys. J. 607, 688 (2004).

[34] M. G. Abadi, J. F. Navarro, M. Steinmetz, and V. R. Eke, Astrophys. J. 591, 499 (2003).

[35] W. J. G. de Blok, S.S. McGaugh, A. Bosma, and V.C. Rubin, Astrophys. J. Lett. 552, L23 (2001).

[36] S. S. McGaugh and W. J. G. de Blok, Astrophys. J. 499, 66 (1998).

[37] S. S. McGaugh, Astrophys. J. 611, 26 (2004).

[38] S. S. McGaugh, Astrophys. J. Lett. 541, L33 (2000).

[39] J. D. Bekenstein, Phys. Rev. D 70, 083509 (2004).

[40] C. Skordis, D. F. Mota, P. G. Ferreira, and C. Boehm, astro-ph/0505519 [Phys. Rev. Lett. (to be published)].

[41] A. Aguirre, J. Schaye, and E. Quataert, Astrophys. J. 561, 550 (2001).

[42] R. H. Sanders, Mon. Not. R. Astron. Soc. 342, 901 (2003).

[43] D. Clowe, A. Gonzalez, and M. Markevitch, Astrophys. J. 604, 596 (2004).

[44] E. Pointecouteau and J. Silk, astro-ph/0505017 (to be published).

[45] R. Scarpa, G. Marconi, and R. Gilmozzi, Astron. Astrophys. 405, L15 (2003).

[46] A. J. Romanowsky, N. G. Douglas, M. Arnaboldi, K. Kuijken, M. R. Merrifield, N. R. Napolitano, M. Capaccioli, and K. C. Freeman, Science 301, 1696 (2003).

[47] A. D. Dolgov and S. H. Hansen, Astropart. Phys. 16, 339 (2002).

[48] G. F. Giudice, E. W. Kolb, A. Riotto, D. V. Semikoz, and I. I. Tkachev, Phys. Rev. D 64, 043512 (2001).

[49] D. N. Spergel and P. J. Steinhardt, Phys. Rev. Lett. 84, 3760 (2000).

[50] F. Piazza and C. Marinoni, Phys. Rev. Lett. 91, 141301 (2003). 